\title{
Research Article \\ Screening Hub Genes of Hepatocellular Carcinoma Based on Public Databases
}

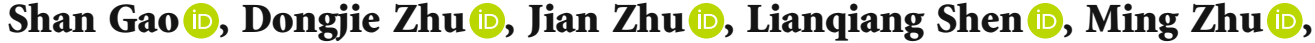 \\ and Xuefeng Ren \\ Department of General Surgery, The First People's Hospital of Yuhang District, Hangzhou, Zhejiang 311100, China \\ Correspondence should be addressed to Xuefeng Ren; jack163163163snow@163.com
}

Received 13 August 2021; Revised 23 September 2021; Accepted 27 September 2021; Published 26 October 2021

Academic Editor: Tao Huang

Copyright (c) 2021 Shan Gao et al. This is an open access article distributed under the Creative Commons Attribution License, which permits unrestricted use, distribution, and reproduction in any medium, provided the original work is properly cited.

\begin{abstract}
Tumor recurrence and metastasis often occur in HCC patients after surgery, and the prognosis is not optimistic. Hence, searching effective biomarkers for prognosis of is of great importance. Firstly, HCC-related data was acquired from the TCGA and GEO databases. Based on GEO data, 256 differentially expressed genes (DEGs) were obtained firstly. Subsequently, to clarify function of DEGs, clusterProfiler package was used to conduct functional enrichment analyses on DEGs. Protein-protein interaction (PPI) network analysis screened 20 key genes. The key genes were filtered via GEPIA database, by which 11 hub genes (F9, CYP3A4, ASPM, AURKA, CDC20, CDCA5, NCAP, PRC1, PTTG1, TOP2A, and KIFC1) were screened out. Then, univariate Cox analysis was applied to construct a prognostic model, followed by a prediction performance validation. With the risk score calculated by the model and common clinical features, univariate and multivariate analyses were carried out to assess whether the prognostic model could be used independently for prognostic prediction. In conclusion, the current study screened HCC prognostic gene signature based on public databases.
\end{abstract}

\section{Introduction}

Liver cancer ranks sixth of most frequent cancers and fourth of primary causes of cancer death throughout the world [1]. Hepatocellular carcinoma (HCC) (accounting for 75\%-85\%) is involved in primary liver cancer [2]. Hepatectomy, radiofrequency ablation, transcatheter arterial chemoembolization, liver transplantation, chemotherapy, and other strategies were applied on HCC, but its prognosis is still not optimistic [3]. About $70 \%$ of HCC patients present tumor metastasis or recurrence within 5 years after surgery [4]. Histological grade, regional invasion, distant metastasis, and other independent risk factors are closely related to recurrence and poor prognosis of HCC [5]. Nevertheless, the continuous improvement of tumor heterogeneity and molecular mechanism research have discovered more and more molecular markers of HCC, which will offer new strategies for HCC treatment.

Microarray technology and bioinformatics methods have been extensively applied to screen differentially expressed genes (DEGs) at the genomic level to help us identify HCC-related DEGs and functional pathways. In addition, gene chips can quickly detect DEGs, generate slice data, and store them in public databases, which is a reliable technique [6]. Therefore, a large number of valuable evidences can be mined for new researches based on these data. For example, more and more potential biomarkers can be mined by using public databases [7-9]. For instance, a study of Wang et al. used RNA sequencing (RNA-seq) data of clear cell renal cell carcinoma from the TCGA database to identify DEGs and 15 hub genes were found to be important in predicting the prognosis and progression of ccRCC [10]. The study of Huang et al. identified the module most related to high-level prostate cancer and revealed the hub genes within the module [11]. For HCC, the top 25\% DEGs from the GSE62232 dataset were selected by Kong et al. to screen modules related to prognosis, and a protein-protein interaction (PPI) network was made to screened out 5 candidate genes including PCNA, RFC4, PTTG1, H2AFZ, and RRM1 [12]. 


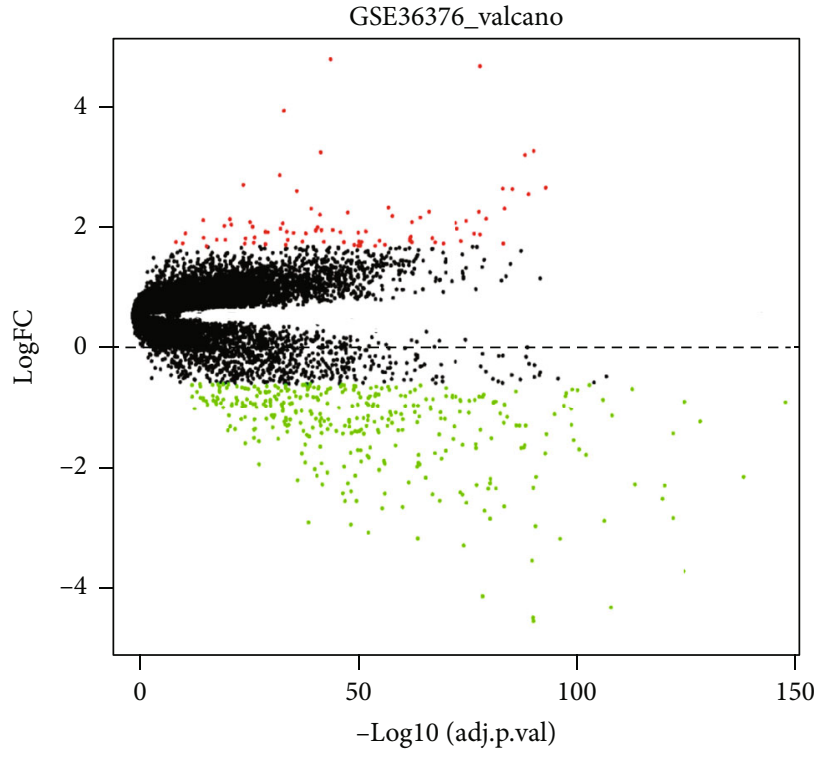

(a)

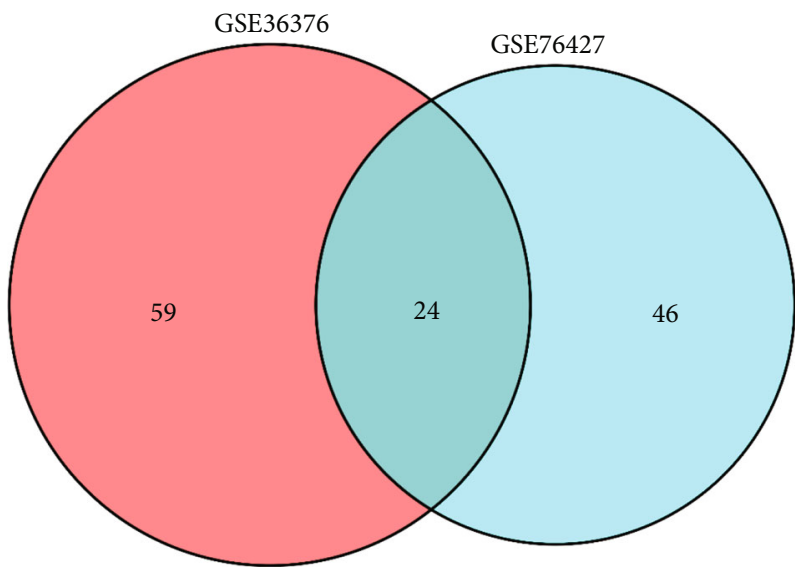

(c)

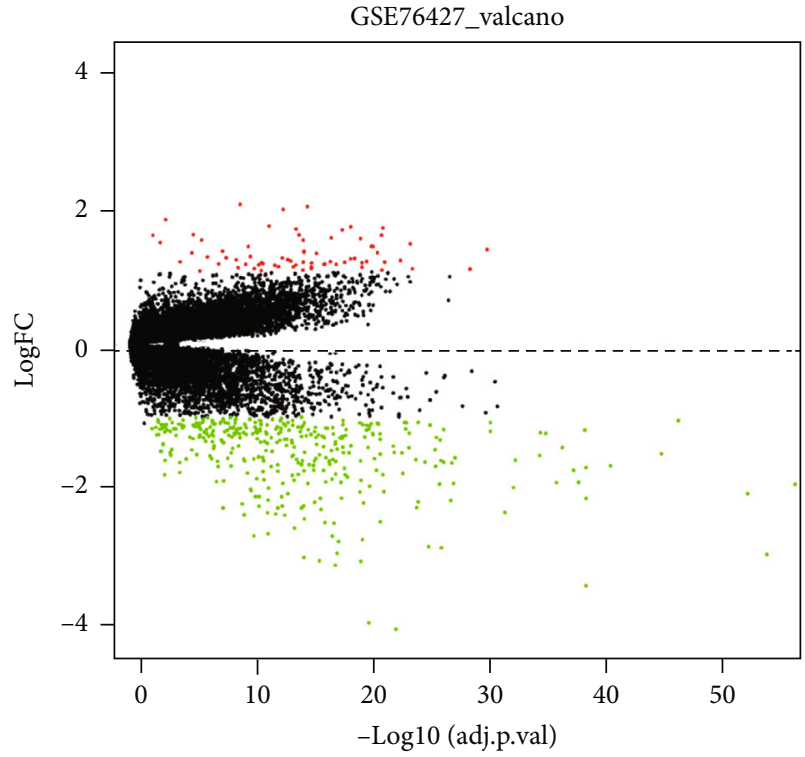

(b)

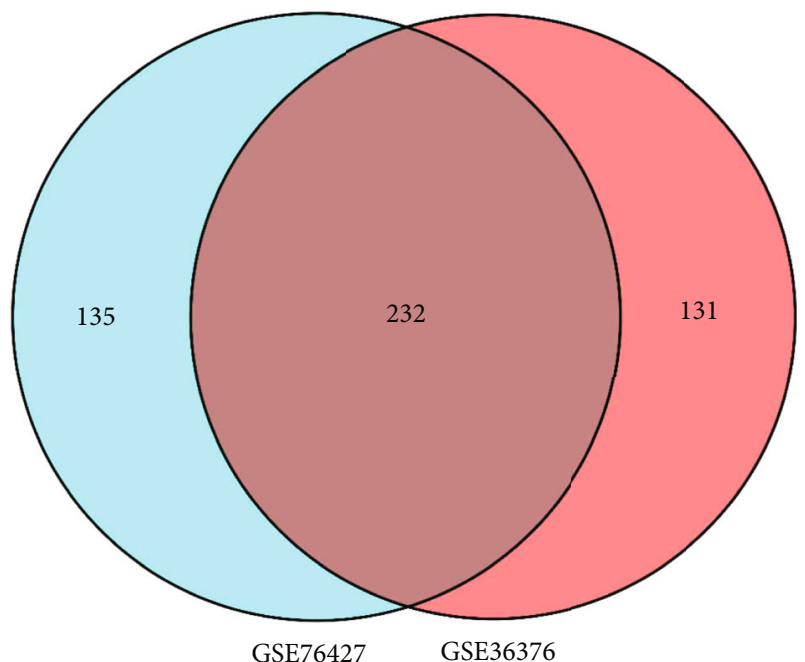

(d)

Figure 1: Identifying significant DEGs. (a, b) Volcano plots of the identified DEGs from GSE36376 and GSE76427. Black dots for nondifferentially expressed genes, and green dots along with red dots for downregulated and upregulated genes, respectively. (c, d) Intersection of upregulated and downregulated DEGs of GSE36376 and GSE76427 datasets.

Here, two mRNA microarray datasets and a seq dataset were obtained from the GEO and TCGA databases; then, DEGs were obtained after analysis. Later, Gene Ontology (GO) and Kyoto Encyclopedia of Genes and Protein interaction (KEGG) enrichment analyses were used to predict the DEGs involved biological functions and pathways. PPI network and Cox analysis were introduced for screening prognostic gene signature.

\section{Materials and Methods}

2.1. Data Download and Processing. GSE36376 (normal 193, tumor 240) and GSE76427 (normal 52, tumor 115) datasets were selected from the GEO database (https://www.ncbi.nlm .nih.gov/geo/). The platform of the both datasets was
GPL10558Illumina HumanHT-12 V4.0 chip. Gene expression matrix and clinical data of HCC were accessed from the TCGA database for further validation. Differential expression analysis $(|\log F C|>1, F D R<0.05)$ was conducted by Limma package [13].

\subsection{Enrichment Analyses of DEGs and PPI Construction. GO} and KEGG enrichment analyses were carried out on DEGs using the clusterProfiler package [14] of R software. GO enrichment analysis is used to study biological significance of DEGs. KEGG pathway enrichment analysis seeks for key pathways closely related to DEGs. Thresholds are as follows: FDR $<0.05$ and $P$ value $<0.05$.

PPI networks were constructed for DEGs by using the STRING database, respectively [15]. Then, interaction 


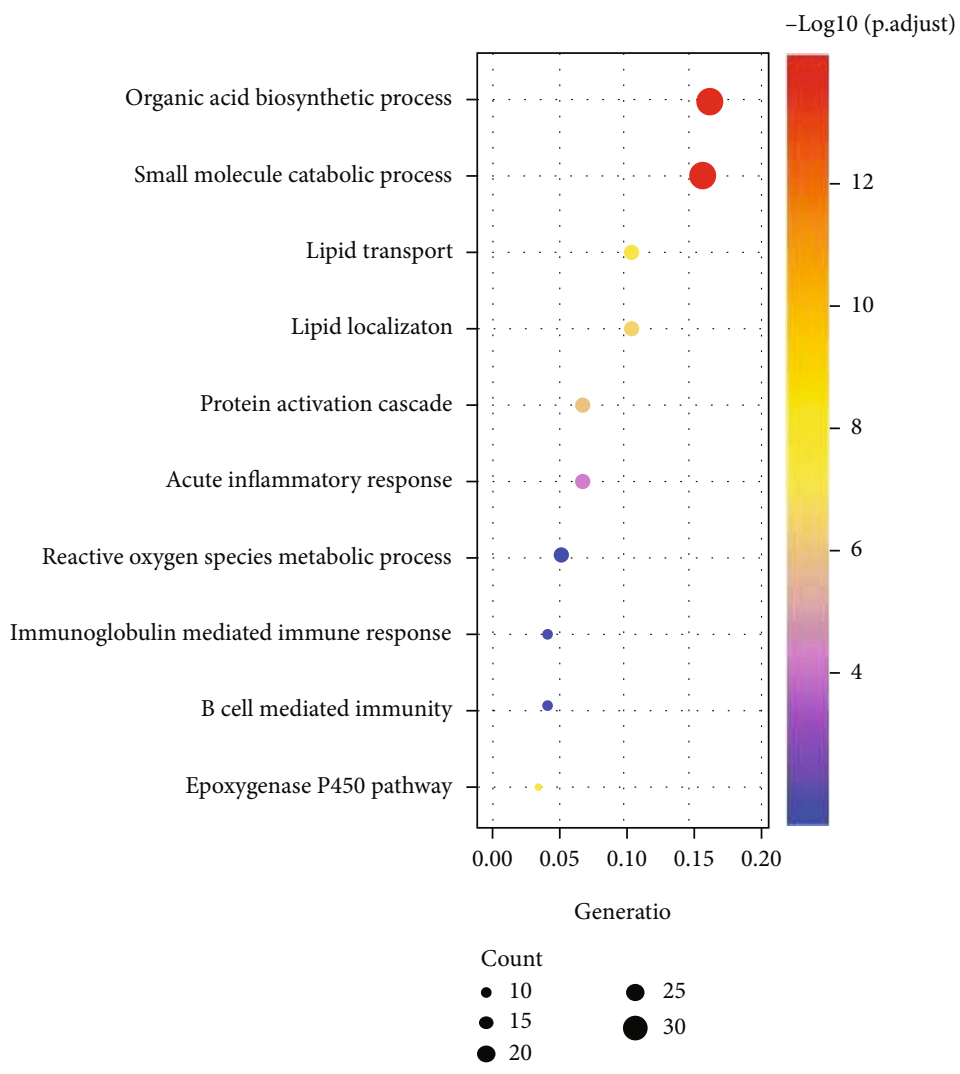

(a)

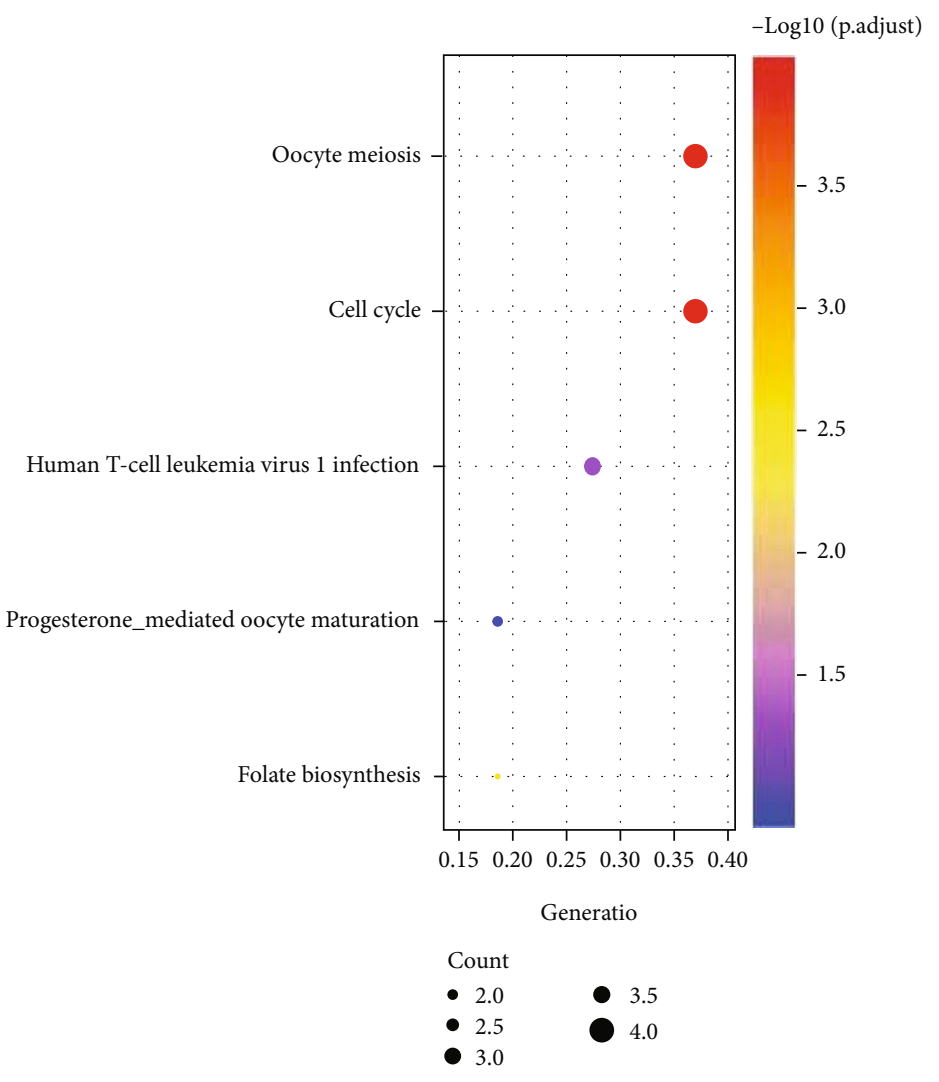

(b)

Figure 2: Continued. 


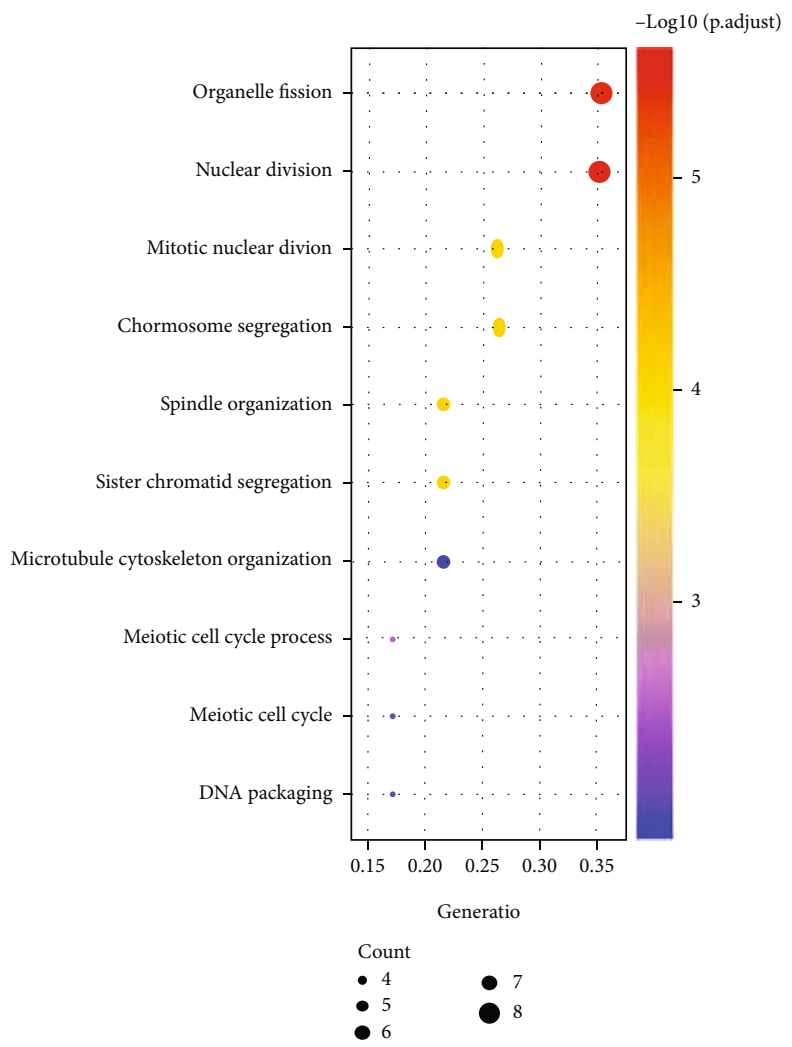

(c)

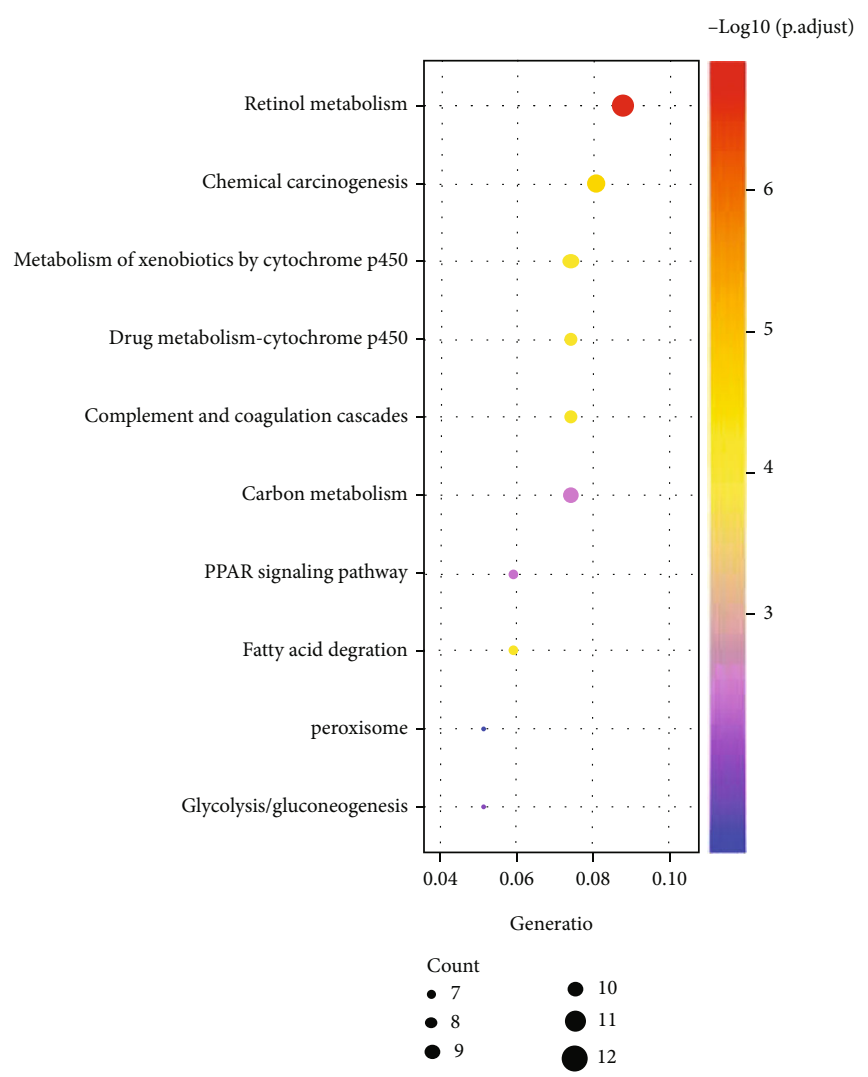

(d)

FIGURE 2: Enrichment analyses for significant DEGs. (a, b) GO and KEGG enrichment analyses for upregulated DEGs. (c, d) GO and KEGG enrichment analyses for downregulated DEGs. 
score $>0.4$ was used to construct PPI networks. Cytoscape 3.7.0 was used to visualize the genes in PPI networks and to exhibit connectivity degree between genes in networks. After that, top ten genes of the number of connection nodes were selected to construct a PPI network diagram separately.

\subsection{Prognostic Model Construction and Evaluation. Cox} regression analysis was applied to construct prognostic model with "survival" R package [16]. The principal component analysis (PCA) was used to determine whether samples could be divided into different clusters based on risk score by the factoextra R package [17]. The ROC curve was plotted by the timeROC package [18].

2.4. Survival Analysis of DEGs. The GEPIA database can be used for individual analysis. In this study, the confirmation of expressions, overall survival (OS) analysis, and diseasefree survival (DFS) analysis of the above key genes were performed using the GEPIA database, and log-rank tests were used to measure statistical significance. Survival R package [16] was used for plotting the survival curve between the high- and low-risk groups.

\section{Results}

3.1. Screening of Important DEGs in HCC. Two datasets (GSE36376 and GSE76427) related to HCC in the GEO database were selected. Subsequently, differential analysis was conducted on the datasets with $|\operatorname{logFC}|>1$ and FDR $<0.05$. There were 446 DEGs in the GSE36376 dataset (83 upregulated DEGs, 363 downregulated DEGs) (Figure 1(a)), while 437 DEGs were in the GSE76427 dataset (70 upregulated DEGs, 367 downregulated DEGs) (Figure 1(b)). Next, DEGs of the two datasets were intersected to obtain the shared DEGs. As shown in Figures 1(c) and 1(d), there were 24 genes of high expression and 232 genes of low expression in common. These important DEGs were selected for subsequent analysis.

3.2. Functional Analyses. GO analysis results revealed that 24 intersected upregulating genes were mainly concentrated in nuclear division, chromosome segregation, mitotic nuclear division, spindle organization, sister chromatid segregation, and other pathways (Figure 2(a)). KEGG analysis exhibited that these genes were mainly gathered in signaling pathways such as oocyte meiosis, cell cycle, and human T-cell virus 1 infection (Figure 2(b)). Intersected downregulating genes were mainly concentrated in small molecule catabolic process, organic acid biosynthetic process, lipid localization, lipid transport, acute inflammatory response pathway, and protein activation cascade (Figure 2(c)). These genes were in the chemical carcinogenesis, retinol metabolism, metabolism of xenobiotics by cytochrome P450, chemical carcinogenesis, complement and coagulation cascades, drug metabolismcytochrome $\mathrm{P} 450$, and carbon metabolism signaling pathways (Figure 2(d)). Therefore, these DEGs may influence the progression of HCC by influencing these pathways.

3.3. Establishment and Analysis of PPI Networks. PPI networks were constructed, and node degree was calculated. The corresponding node degrees of top 10 hub genes were
TABle 1: Top 10 hub genes in PPI networks.

\begin{tabular}{lc}
\hline Gene symbol & Degree \\
\hline Upregulated genes & \\
ASPM & 12 \\
AURKA & 12 \\
CCNB2 & 12 \\
CDC20 & 12 \\
CDCA5 & 12 \\
NCAPG & 12 \\
PRC1 & 12 \\
PTTG1 & 12 \\
TOP2A & 12 \\
KIFC1 & 11 \\
Downregulated genes & \\
FTCD & 25 \\
C8A & 23 \\
F9 & 23 \\
CYP3A4 & 22 \\
GPT & 21 \\
APOA5 & 20 \\
FETUB & 20 \\
MBL2 & 20 \\
KLKB1 & 19 \\
SPP2 & 19 \\
\hline
\end{tabular}

exhibited in Table 1. In Figures 3(a) and 3(b), PPI networks with upregulated and downregulated significant bias were, respectively, constructed. For better visualization, Cytoscape was used to construct the interaction diagrams of 10 highly expressed and 10 lowly expressed hub genes. It could be observed that there were interactions between these genes, indicating that the interactions may be associated with development of HCC (inhibiting or promoting the development of HCC). In addition, TOP2A and FTCD were located in the center of the diagram, so it was considered that they could be used as targets to further explore their role in HCC.

3.4. Hub Gene Identification and Prognostic Model Construction in HCC. 20 key genes were analyzed by the GEPIA database, and it was suggested that the upregulation and downregulation of 15 genes were in accordance with this study. Both the OS curves of 13 genes and the DFS of 14 genes had biological significance. Genes with significant differences in expression analysis, OS analysis, and DFS analysis were intersected, and 11 genes (ASPM, AURKA, CDC20, CDCA5, KIFC1, NCAPG, PRC1, PTTG1, TOP2A, CYP3A4, and F9) were finally screened out as hub genes for subsequent verification (Figure 4(a)). To examine the correlation between the hub genes and prognosis status, univariate Cox analysis was introduced constructing a 10-gene prognostic signature in the TCGA dataset which was referred to as the training set (Figure 4(b)). The samples from the TCGA database were divided into the high- and low-risk groups based on the median value of risk score (Figure 4(c)). Also, the expression profile and survival status 


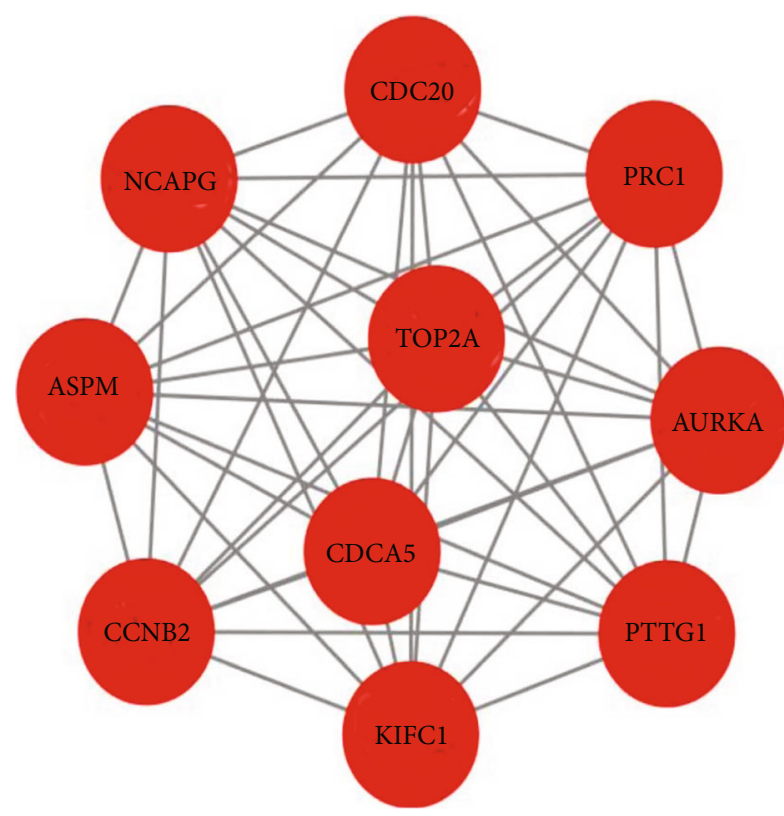

(a)

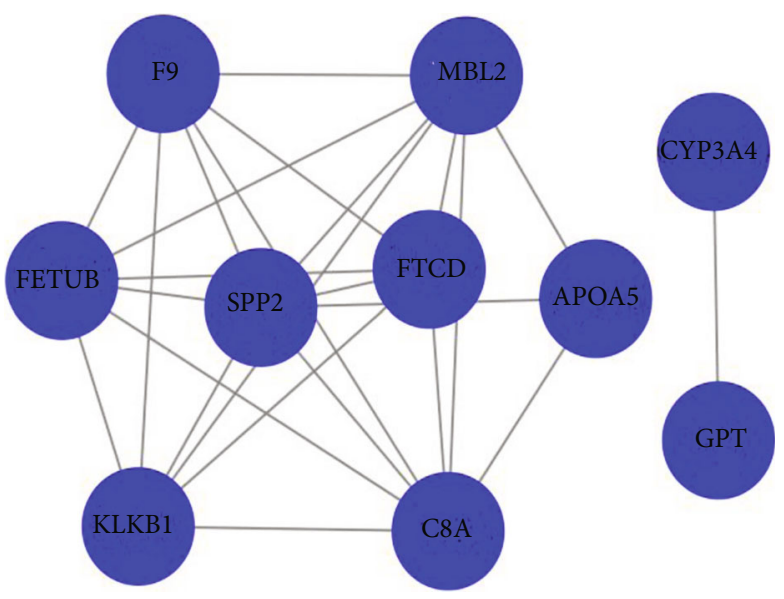

(b)

Figure 3: Top 10 hub genes in PPI networks. (a, b) Top 10 hub genes (depending on node degree) of significant highly expressed and lowly expressed DEGs, respectively. Node degree indicates the importance of genes (nodes) in the PPI network.

distribution of the samples were presented (Figures 4(d) and $4(\mathrm{e})$ ). To examine the prediction performance, ROC curves was plotted based on the training set and validation set (GSE76427), respectively, revealing an optimal performance in the predictions (Figures 4(f) and 4(g)). In addition, PCA and survival analysis were employed for a further validation. PCA indicated that the high- and low-risk groups were obviously divided into two clusters (Figure 4(h)), and for survival analysis, patients with low risk shared a relatively optimal survival status (Figure 4(i)). To identify whether risk score could be used as an independent risk indicator, univariate and multivariate Cox analyses were conducted on the common clinical features and risk score. As the results illustrated, the prognostic model presented a robust independency (Figures 5(a) and 5(b)). Moreover, a nomogram based on the clinical features and risk score was designed for a comprehensive prediction of 1-, 3-, and 5-year-survival rates, followed by plotting the calibration curves between actual and predicted survival rates (Figures $5(\mathrm{c})-5(\mathrm{f})$ ).

3.5. Examination of the Prognosis-Related Genes. To verify the prognosis-related genes (ASPM, AURKA, CDC20, CDCA5, KIFC1, NCAPG, PRC1, PTTG1, TOP2A, and CYP3A4) in HCC, the expression analysis between tumor and normal samples were presented, where we observed that the mRNA expressions of all the genes except for CYP3A4 were significantly upregulated, while CYP3A4 presented the opposite trend (Figure 6). Then, survival analyses between the high and low expressions of the genes were performed, where high expression of all the genes except for CYP3A4 gave rise to poor prognostic performances (Figures 7 and 8). To summarize, 10 prognosis-related genes showed significant difference in the terms of expression and prognostic performance.

\section{Discussion}

Main causes of HCC include chronic hepatitis virus infection, gene mutation, cell damage, alcoholic liver disease, and aflatoxin poisoning. But molecular mechanism of HCC is still less studied. An important role in HCC is cell cycle regulator [19-21]. Our study also demonstrates that the functional enrichment of DEGs is significantly upregulated in the cell cycle pathway. Cyclin D1, c-myc, RAS mutations, and cyclin D2 promoter hypermethylation are associated with HCC $[22,23]$. Moreover, splicing changes of NT5E, Sulf1, and SLC39A14 were also associated with HCC [24-26]. Most HCC patients without early detection are not suitable for radical treatment, which may lead to poor prognosis of patients. Hence, potential as well as efficient markers are in urgent need. Microarray technology helps us to investigate genetic changes in HCC and identify novel biomarkers in other diseases.

Herein, DEGs were obtained from two datasets. 883 DEGs include 153 highly expressed genes and 730 lowly expressed genes. Enrichment analyses exhibited that genes with high expression were enriched in nuclear fission, mitosis, cell cycle, DNA packaging, oocyte meiotic division, folic acid synthesis, and the oocyte maturation of progesterone-mediated pathway. Lowly expressed genes were mainly in metabolic process, main immune pathways, and oxidation process, such as organic acid biosynthesis, small molecule catabolism process, lipid transport and positioning, immunoglobulin-mediated immune response, B cell-mediated response, cyclooxygenase, and P450 pathways. Cell cycle process dysregulation and mitotic cell cycle are vital in tumor development $[23,27,28]$. CDC20, one of the cell cycle regulators, was reported to serve as an oncogene [29], and in the latest study, the tumorigenesis role 


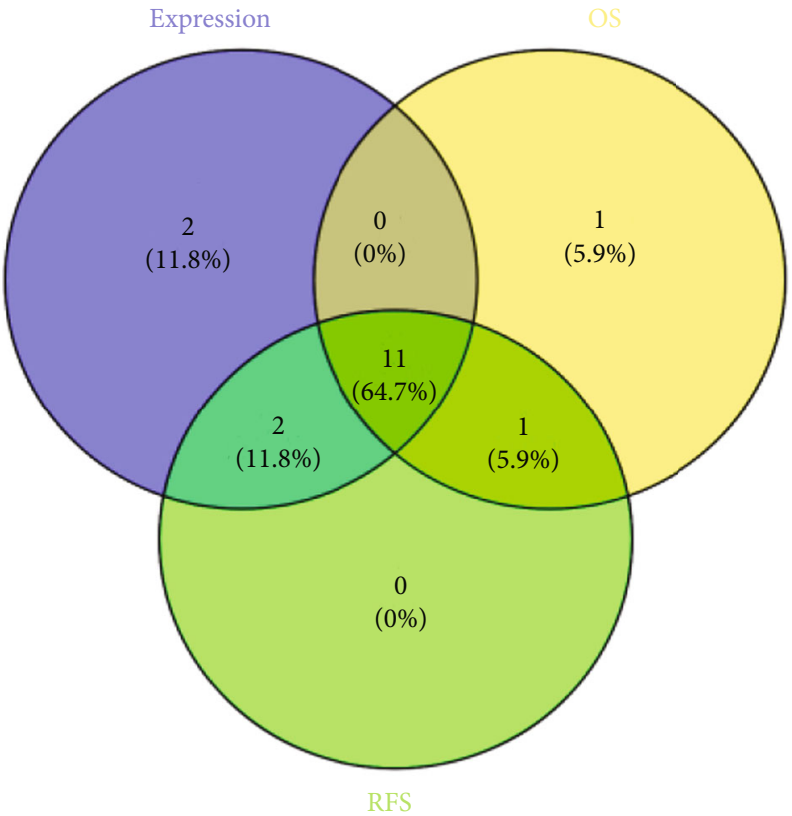

(a)

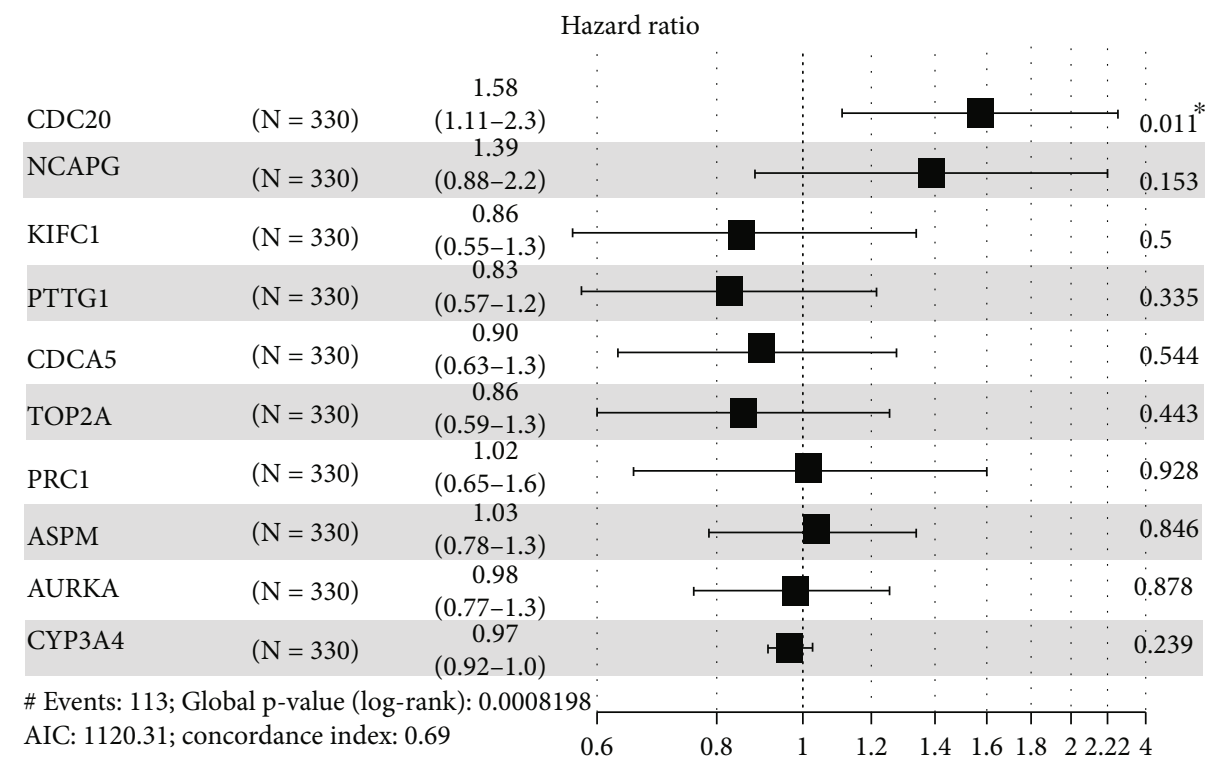

(b)

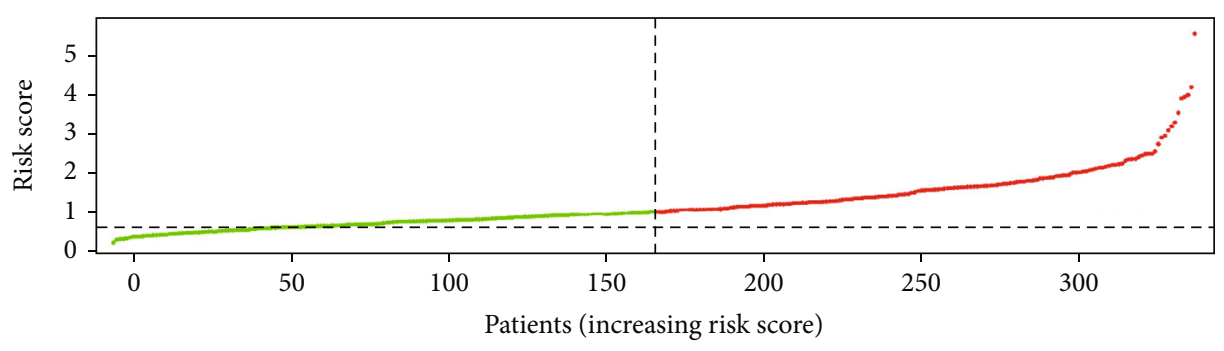

(c)

FIgURe 4: Continued. 


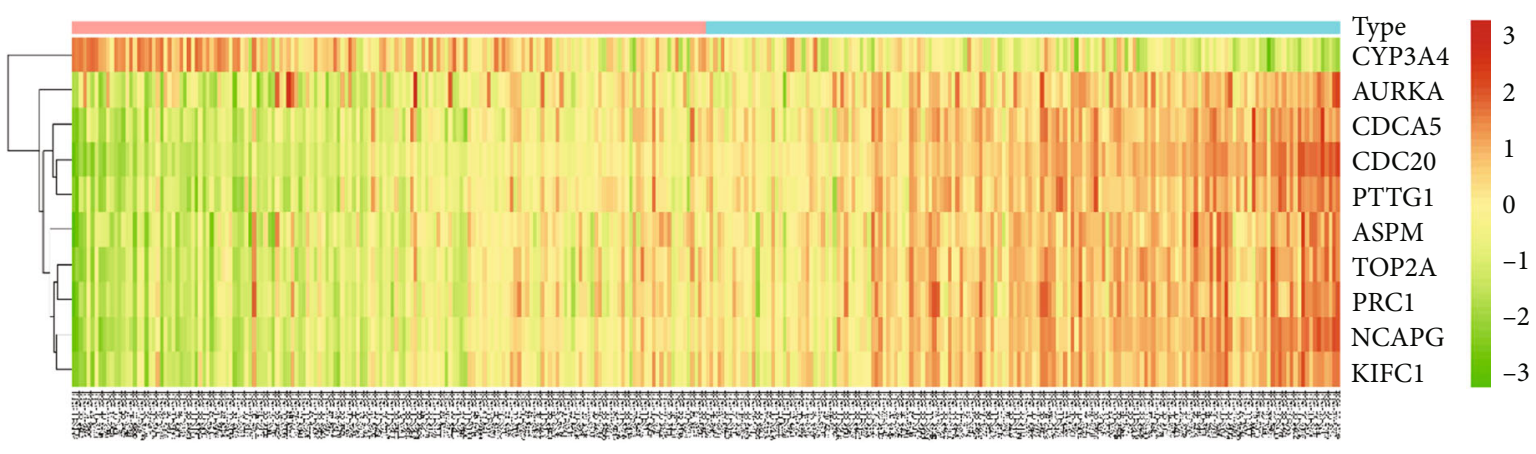

Type

High

Low

(d)

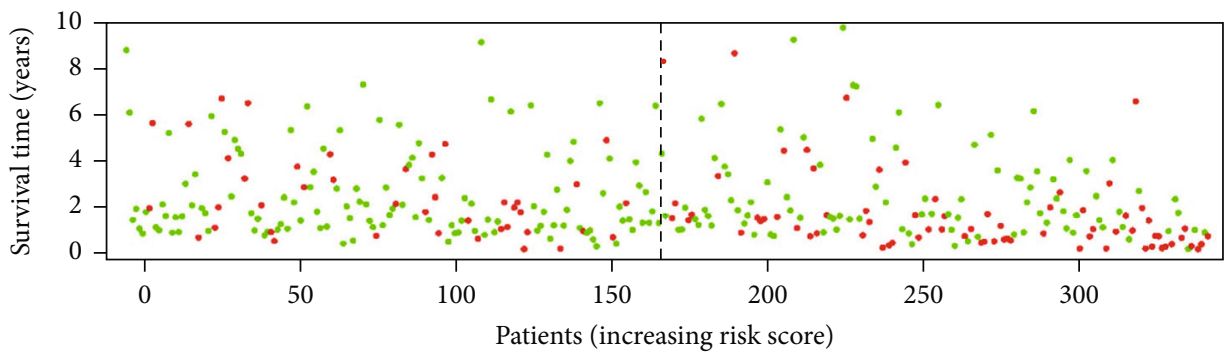

- Dead

- Alive

(e)
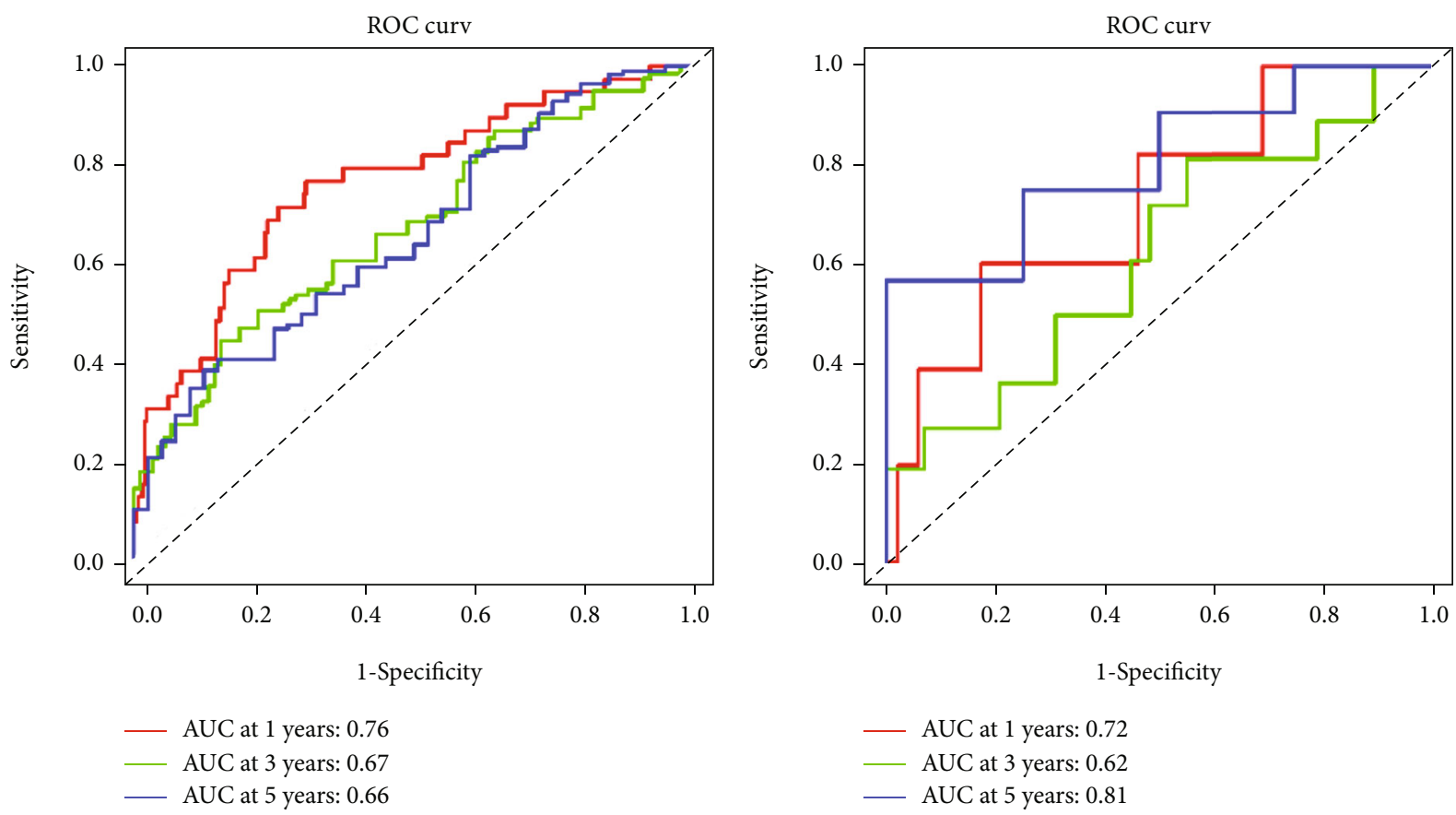

(f)

(g)

Figure 4: Continued. 


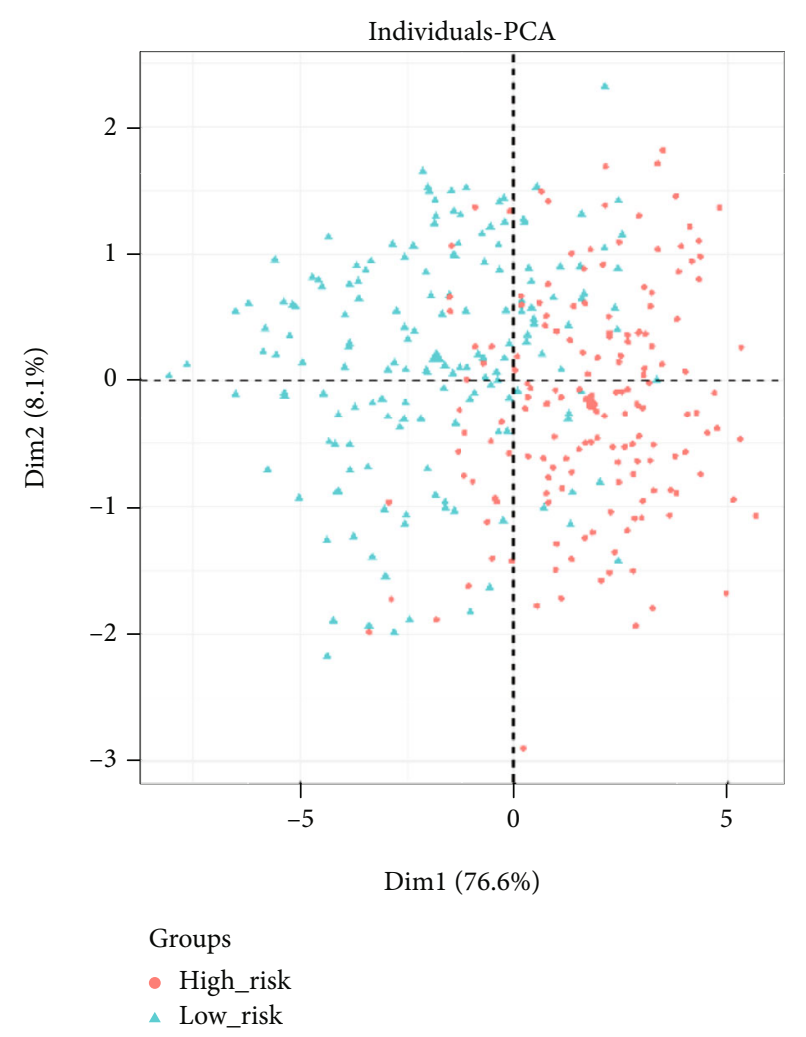

(h)

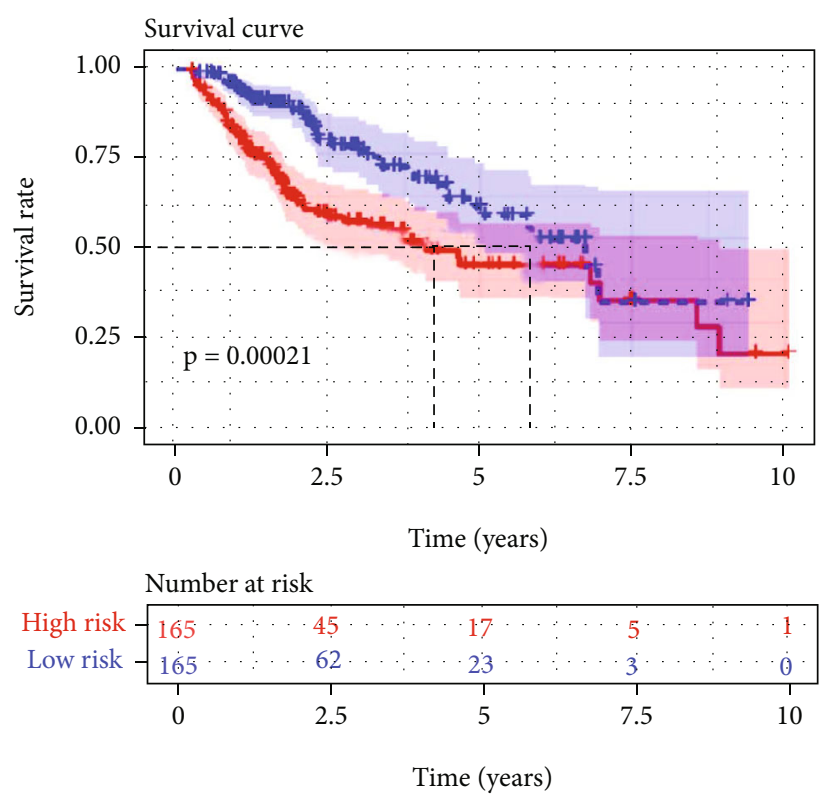

Strata
+ High risk
+ Low risk

FIGURE 4: Screening of hub genes and constructing prognostic model. (a) Identification of hub genes, OS and DFS analyses using GEPIA. (b) Univariate cox analysis for the hub genes. (c) Arrange the samples on risk score. (d) Heat map of prognostic gene signature between the high- and low-risk groups. (e) Distribution of samples on risk score. (f, g) ROC curve of prognostic signature on 1-, 3-, and 5-year OS in the training and validation sets, respectively. (h) PCA for high- and low-risk samples (I) K-M analysis for the high- and low-risk groups; ${ }^{*} P<0.05$.

and the molecular mechanism of CDC20 in HCC development was pointed out as well [30]. In conclusion, GO enrichment analysis indicated that changes were mainly gathered in cell division, nuclear division, and mitosis. Changes of KEGG were in chemical carcinogenesis, glycolysis/gluconeogenesis, drug-cytochrome P450, complement and coagulation cascade, carbon metabolism, PPAR, and other signaling pathways.

In the PPI network diagrams, we selected 10 highly expressed genes and 10 lowly expressed genes as hub genes, with node degree greater than 10. Among these hub genes, the node degree of upregulated ASPM, AURKA, CCNB2, CDC20, CDCA5, NCAPG, PRC1, PTTG1, and TOP2A was 12, while the node degree of downregulated FTCD was up to 25. In these hub genes, TOP2A is confirmed to be related to early onset of HCC, shorter survival, microvascular invasion, chemotherapy resistance, and recurrence [31, 32]. Hence, it is considered a target for anticancer drugs [33-35]. HER2 and TOP2A are usually coamplified in HER2-amplified breast cancer [36]. However, the overexpression of TOP2A in HCC was not correlated with the overexpression of HER2 [37]. Besides, TOP2A can be a biomarker for diagnosis, treatment, and prognosis in lung cancer, colon cancer, and ovarian cancer [38-40]. Some clinical reports have indicated that overexpressing TOP2A is remarkably associated with shorter survival time [35, 37].
Formiminotransferase cyclodeaminase (FTCD) is expressed in every mammal, but its accumulation is highest in the liver [41]. FTCD contains two active sites (FT and CD) in different protein structures and catalyzes histidine degradation during folate metabolism [42]. Furthermore, FTCD is involved in the Golgi complex and metabolic processes [43]. FTCD is considered a candidate tumor inhibitor in HCC, which inhibits HCC by regulating cell apoptosis, DNA damage, and the phosphatidylinositol 3-kinase/Akt signaling pathway. Overexpression of FTCD inhibits cell proliferation in HCC, resulting in increased PTEN protein level in HCC cells but decreased PI3K, total Akt, and phosphorylated Akt protein levels [44]. In HCC, FTCD can also serve as a useful diagnostic biomarker to distinguish early HCC and benign tumors [45]. Herein, PPI networks showed that both TOP2A and FTCD were in the central position and had direct or indirect interactions with other genes, indicating that TOP2A and FTCD played a key role in HCC development. Later, the GEPIA database analyzed the 20 hub genes combined with expression analysis, OS analysis, and DFS analysis, and finally, 11 genes were selected for the prognostic model construction. Most of them are involved in the development of HCC and can be used as prognostic markers of HCC. An example is that upregulation of CDC20 may predict decreased OS and DFS in HCC patients [46], which is in accordance with our findings. Above 


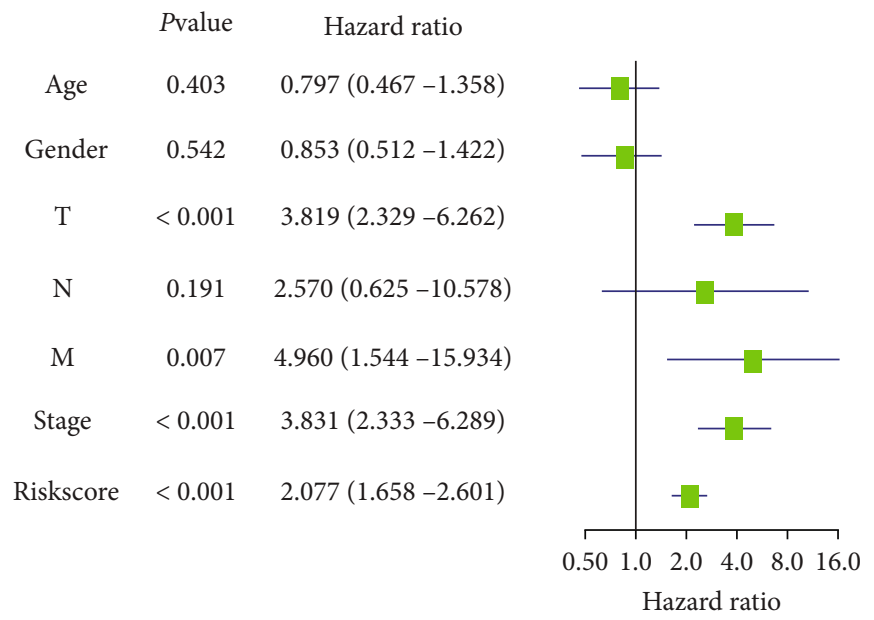

(a)

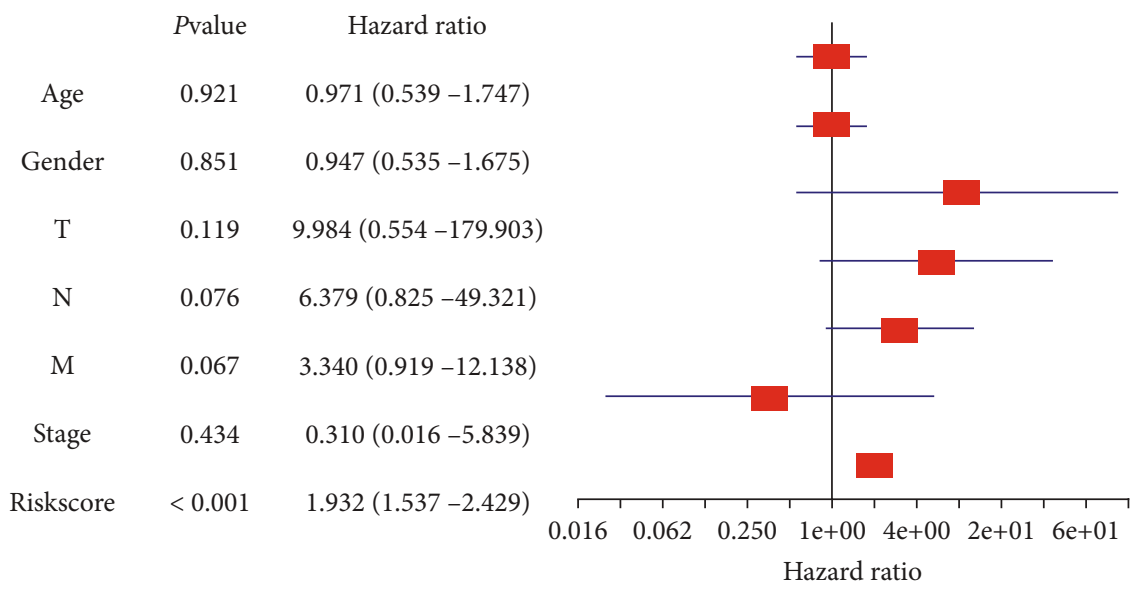

(b)

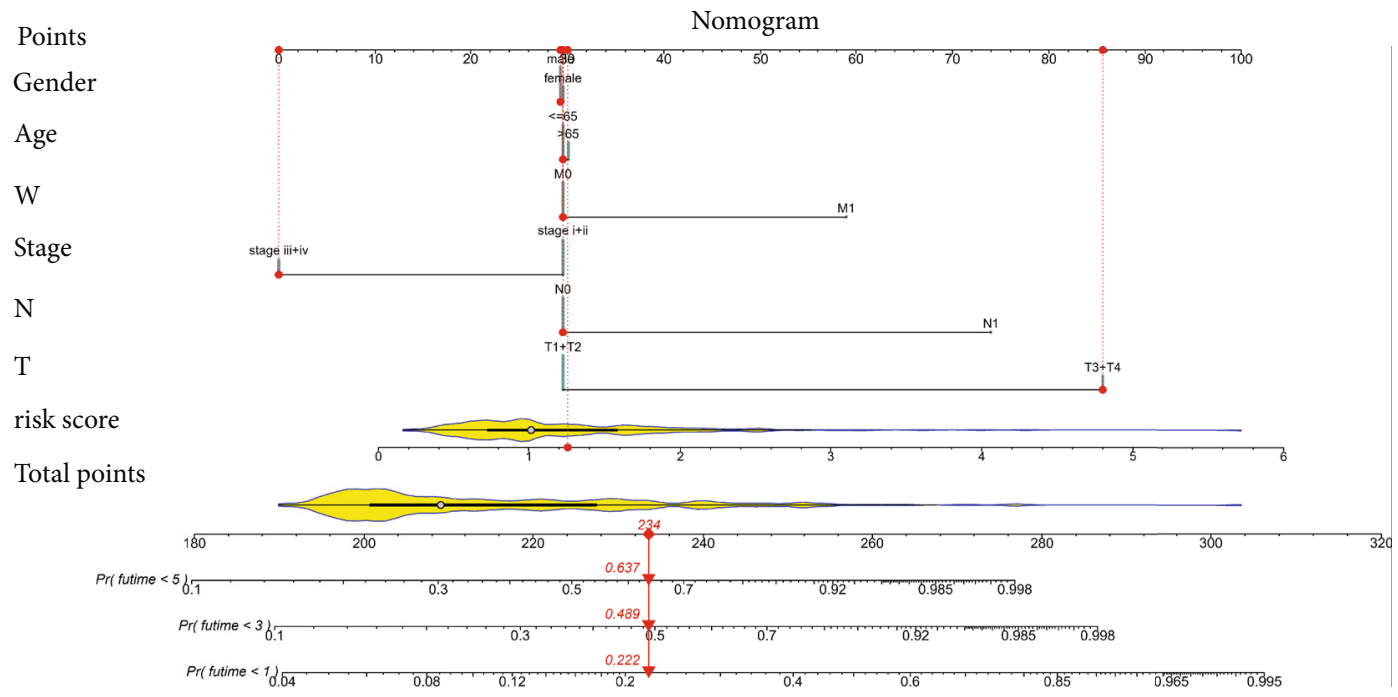

(c)

FIgURE 5: Continued. 


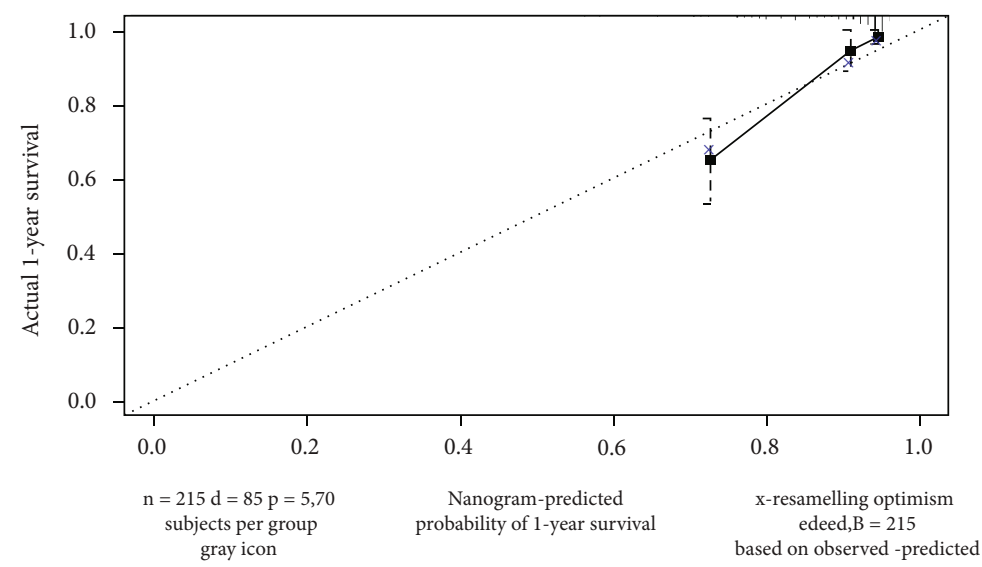

(d)

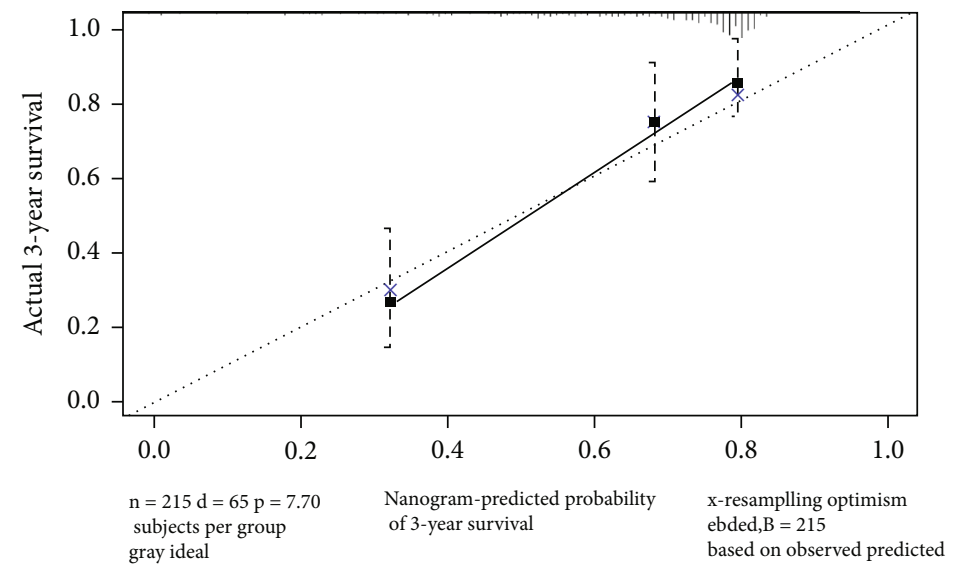

(e)

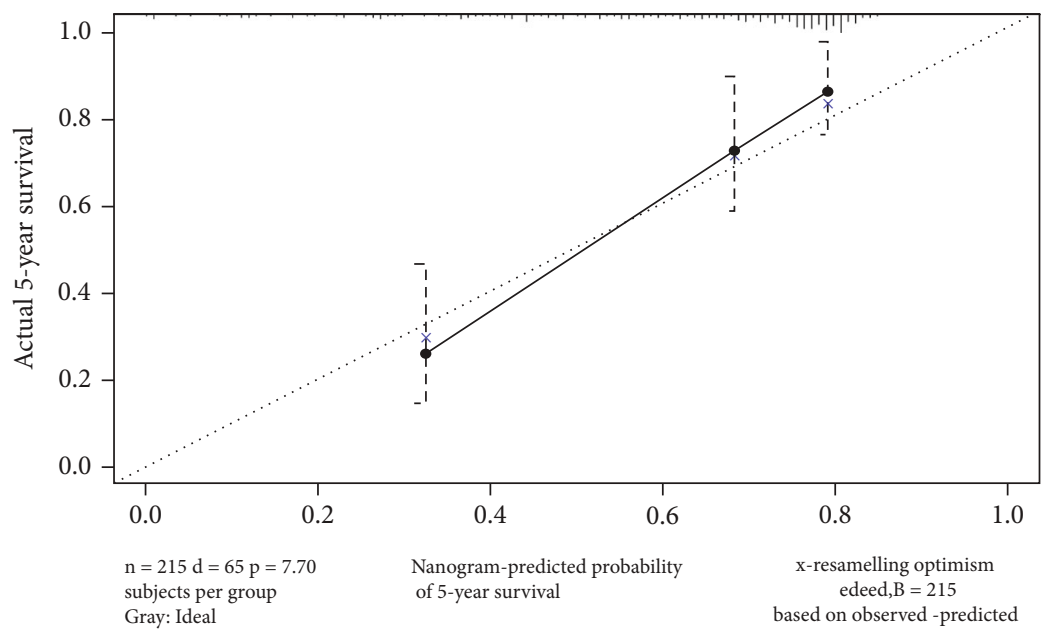

(f)

Figure 5: Assessment of the prognostic model. $(\mathrm{a}, \mathrm{b})$ Univariate and multivariate Cox regression analyses between the risk score and clinical features. (c) Nomogram on clinical features and risk score. (d-f) Calibration curves for the predicted survival status; ${ }^{*} P<0.05,{ }^{* *} P<0.01$, ${ }^{* * *} P<0.001$, and ${ }^{* * * *} P<0.0001$. 


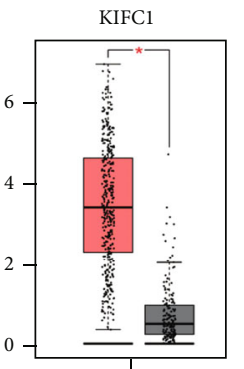

LHC $(\operatorname{num}(\mathrm{T})=369 ; \operatorname{num}(\mathrm{N})=160)$

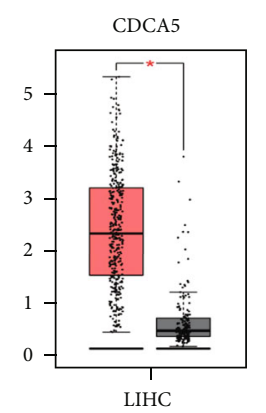

$(\operatorname{num}(\mathrm{T})=369 ; \operatorname{num}(\mathrm{N})=160)$

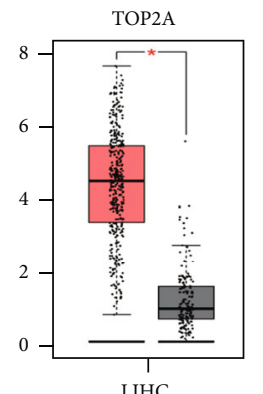

$(\operatorname{num}(\mathrm{T})=369 ; \operatorname{num}(\mathrm{N})=160)$

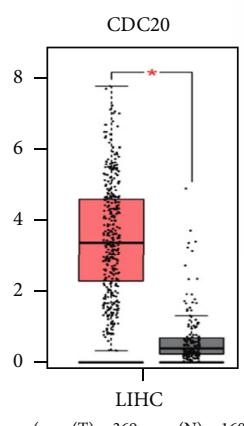

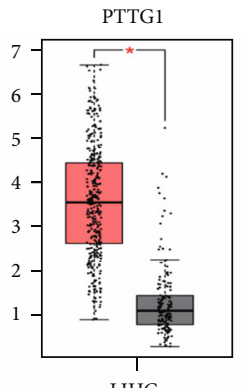

$(\operatorname{num}(\mathrm{T})=369 ; \operatorname{num}(\mathrm{N})=160)$

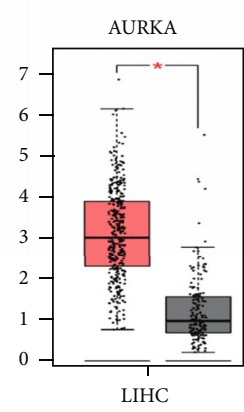

$(\operatorname{num}(\mathrm{T})=369 ; \operatorname{num}(\mathrm{N})=160)$

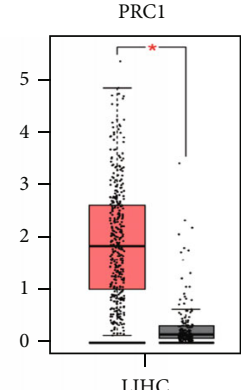

$(\operatorname{num}(\mathrm{T})=369 ; \operatorname{num}(\mathrm{N})=160)$

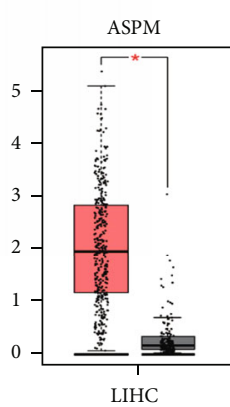

$(\operatorname{num}(\mathrm{T})=369 ; \operatorname{num}(\mathrm{N})=160$

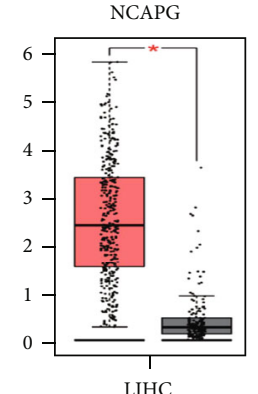

$(\operatorname{num}(\mathrm{T})=369 ; \operatorname{num}(\mathrm{N})=160)$

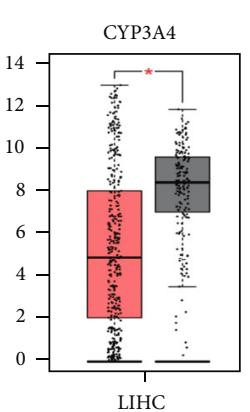

$(\operatorname{num}(\mathrm{T})=369 ; \operatorname{num}(\mathrm{N})=160$

FIGURE 6: Expression status of the prognosis-related genes.
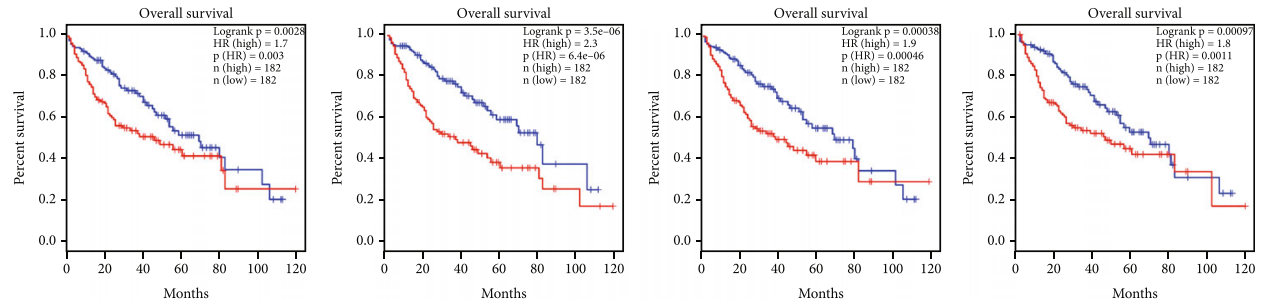

$$
\begin{aligned}
& \text { - Low TOP2A TPM } \\
& \text { - High TOP2A TPM }
\end{aligned}
$$

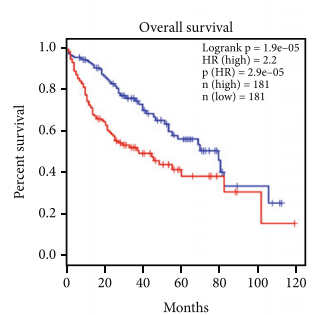

$$
\begin{aligned}
& \text { - Low PTTG1 TPM } \\
& \text { - High PTTG1 TPM }
\end{aligned}
$$

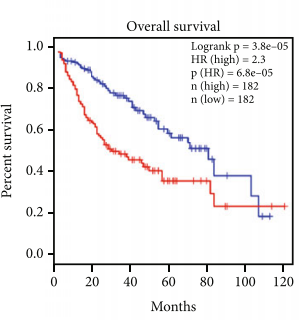

$$
\begin{aligned}
& \text { - Low PRC1 TPM } \\
& \text { - High PRC1 TPM }
\end{aligned}
$$

$$
\begin{aligned}
& \text { - } \\
& \text { - How NCAPG TPM } \\
& \text { High NCAPG TPM }
\end{aligned}
$$
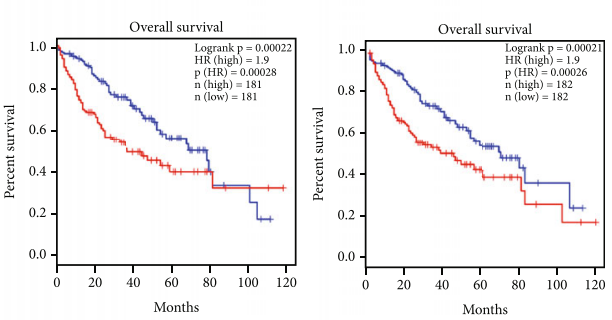

$$
\begin{aligned}
& \text { - Low KIFC1 TPM } \\
& \text { - High KIFC1 TPM }
\end{aligned}
$$

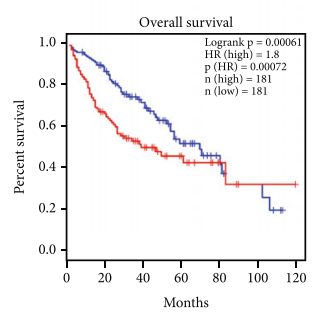

- Low CDC20 TPM
- High CDC20 TPM

- Low AURKA TPM
- High AURKA TPM

- Low CDCA5 TPM
- High CDCA5 TPM

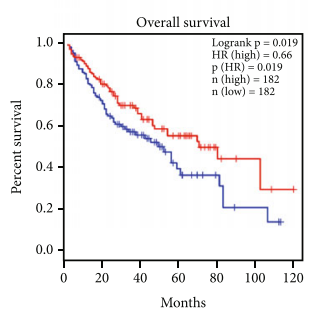

- Low ASPM TPM

- Low CYP3A4 TPM
- High CYP3A4 TPM

FIGURE 7: OS survival analysis depending on the expression of prognosis-related genes. 

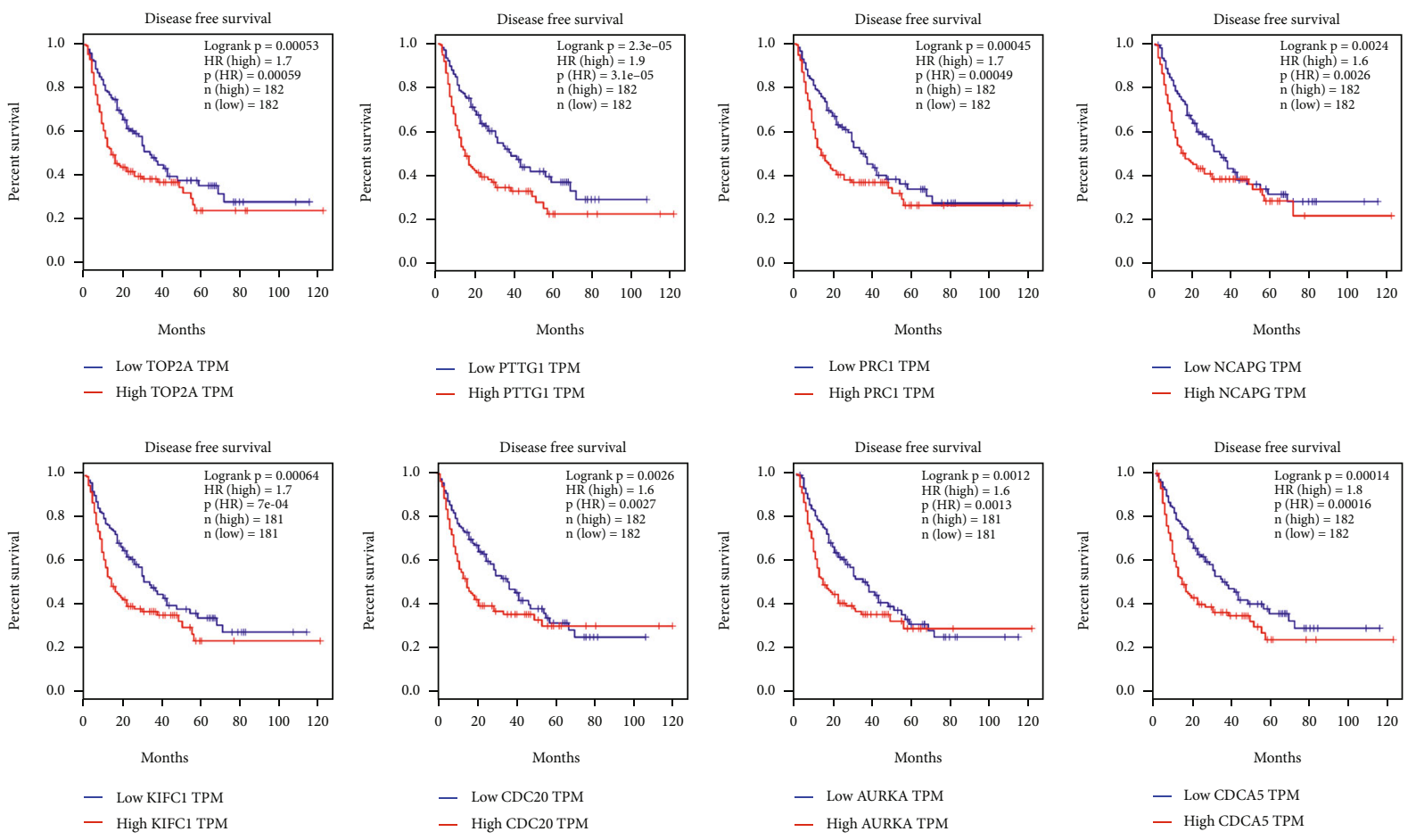

$$
\begin{array}{ll}
\text { - } & \text { Low PRC1 TPM } \\
\text { - } & \text { High PRC1 TPM }
\end{array}
$$

— Low NCAPG TPM - High NCAPG TPM
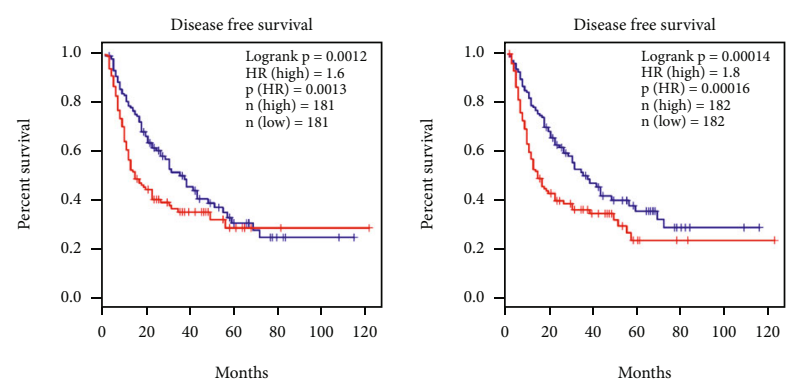

- Low AURKa TPM

- Low CDCA5 TPM
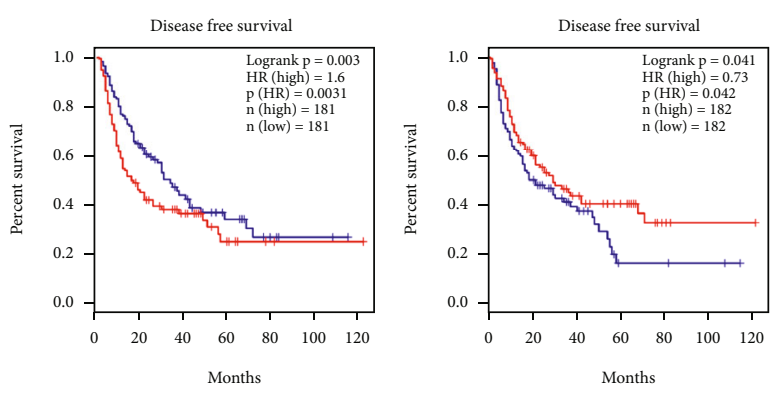

$\begin{array}{ll}\text { - } & \text { Low ASPM TPM } \\ \text { - } & \text { High ASPM TPM }\end{array}$

- Low CYP3A4 TPM

- High CYP3A4 TPM

FIGURE 8: DFS survival analysis depending on the expression of prognosis-related genes.

studies fully demonstrate the importance of these hub genes in HCC progression.

To extract prognosis-related genes among the 20 genes from PPI network, Cox regression analysis was applied, whereby a 10-gene prognostic signature was constructed. As followed, a validation process was conducted by ROC, K-M, and PCA. Several studies have presented HCC prognostic signatures following the similar strategy $[47,48]$. However, compared to the above studies, we performed a more robust HCC prognostic model based on ROC analysis results.

In summary, combining the GEO and TCGA datasets, we screened HCC prognosis-related genes, followed by examination for the prognostic model. However, the corresponding wet experiments which we are designing have not been arranged yet for practical validation.

\section{Data Availability}

The data that support the findings of this research are available on reasonable request from the corresponding author.

\section{Conflicts of Interest}

The authors declare that they have no conflicts of interest.

\section{References}

[1] H. B. El-Serag and K. L. Rudolph, "Hepatocellular carcinoma: epidemiology and molecular carcinogenesis," Gastroenterology, vol. 132, no. 7, pp. 2557-2576, 2007.

[2] F. Bray, J. Ferlay, I. Soerjomataram, R. L. Siegel, L. A. Torre, and A. Jemal, "Global cancer statistics 2018: GLOBOCAN estimates of incidence and mortality worldwide for 36 cancers in 185 countries," CA: a Cancer Journal for Clinicians, vol. 68, no. 6, pp. 394-424, 2018.

[3] A. Villanueva, Y. Hoshida, C. Battiston et al., "Combining clinical, pathology, and gene expression data to predict recurrence of hepatocellular carcinoma," Gastroenterology, vol. 140, no. 5, pp. 1501-1512.e2, 2011.

[4] F. Ji, Y. Liang, S. Fu et al., "Prognostic value of combined preoperative prognostic nutritional index and body mass index in HCC after hepatectomy," HPB: The Official Journal of the 
International Hepato Pancreato Biliary Association, vol. 19, no. 8, pp. 695-705, 2017.

[5] K. Ikeda, S. Saitoh, A. Tsubota et al., "Risk factors for tumor recurrence and prognosis after curative resection of hepatocellular carcinoma," Cancer, vol. 71, no. 1, pp. 19-25, 1993.

[6] B. Vogelstein, N. Papadopoulos, V. E. Velculescu, S. Zhou, L. A. Diaz Jr., and K. W. Kinzler, "Cancer genome landscapes," Science, vol. 339, no. 6127, pp. 1546-1558, 2013.

[7] D. Huang, W. Sun, Y. Zhou et al., "Mutations of key driver genes in colorectal cancer progression and metastasis," Cancer Metastasis Reviews, vol. 37, no. 1, pp. 173-187, 2018.

[8] B. $\mathrm{Li}, \mathrm{K} . \mathrm{Pu}$, and $\mathrm{X}$. Wu, "Identifying novel biomarkers in hepatocellular carcinoma by weighted gene co-expression network analysis," Journal of Cellular Biochemistry, vol. 120, no. 7, pp. 11418-11431, 2019.

[9] E. Martinez-Ledesma, R. G. Verhaak, and V. Treviño, "Identification of a multi-cancer gene expression biomarker for cancer clinical outcomes using a network-based algorithm," Scientific Reports, vol. 5, no. 1, p. 11966, 2015.

[10] Y. Wang, L. Chen, G. Wang et al., "Fifteen hub genes associated with progression and prognosis of clear cell renal cell carcinoma identified by coexpression analysis," Journal of Cellular Physiology, vol. 234, no. 7, pp. 10225-10237, 2019.

[11] H. Huang, Q. Zhang, C. Ye et al., "Identification of prognostic markers of high grade prostate cancer through an integrated bioinformatics approach," Journal of Cancer Research and Clinical Oncology, vol. 143, no. 12, pp. 2571-2579, 2017.

[12] J. Kong, T. Wang, Z. Zhang, X. Yang, S. Shen, and W. Wang, "Five core genes related to the progression and prognosis of hepatocellular carcinoma identified by analysis of a coexpression network," DNA and Cell Biology, vol. 38, no. 12, pp. 1564-1576, 2019.

[13] M. E. Ritchie, B. Phipson, D. Wu et al., "Limma powers differential expression analyses for RNA-sequencing and microarray studies," Nucleic Acids Research, vol. 43, no. 7, p. e47, 2015.

[14] G. Yu, L. G. Wang, Y. Han, and Q. Y. He, "clusterProfiler: an R package for comparing biological themes among gene clusters," OMICS, vol. 16, no. 5, pp. 284-287, 2012.

[15] D. Szklarczyk, A. Franceschini, M. Kuhn et al., "The STRING database in 2011: functional interaction networks of proteins, globally integrated and scored," Nucleic Acids Research, vol. 39, no. Database, pp. D561-D568, 2011.

[16] T. M. Therneau and P. M. Grambsch, Modeling Survival Data: Extending the Cox Model, Springer, 2000.

[17] B. Nowak, A. Mucha, M. Moska, and W. Kruszyński, "Reproduction indicators related to litter size and reproduction cycle length among sows of breeds considered maternal and paternal components kept on medium-size farms," Animals (Basel), vol. 10, no. 7, p. 1164, 2020.

[18] P. Blanche, J. F. Dartigues, and H. Jacqmin-Gadda, "Estimating and comparing time-dependent areas under receiver operating characteristic curves for censored event times with competing risks," Statistics in Medicine, vol. 32, no. 30, pp. 5381-5397, 2013.

[19] X. H. Liang, Z. P. Feng, F. Q. Liu et al., "MAPRE1 promotes cell cycle progression of hepatocellular carcinoma cells by interacting with CDK2," Cell Biology International, vol. 44, no. 11, pp. 2326-2333, 2020.

[20] Z. Liu, J. Li, J. Chen et al., "MCM family in HCC: MCM6 indicates adverse tumor features and poor outcomes and promotes
S/G2 cell cycle progression," BMC Cancer, vol. 18, no. 1, p. 200, 2018.

[21] L. Tuo, J. Xiang, X. Pan et al., "PCK1 negatively regulates cell cycle progression and hepatoma cell proliferation via the AMPK/p27Kip1 axis," Journal of Experimental \& Clinical Cancer Research, vol. 38, no. 1, p. 50, 2019.

[22] M. Gorath, T. Stahnke, T. Mronga, O. Goldbaum, and C. Richter-Landsberg, "Developmental changes of tau protein and mRNA in cultured rat brain oligodendrocytes," Glia, vol. 36, no. 1, pp. 89-101, 2001.

[23] Y. Wang, J. Cheng, C. Xu et al., "Quantitative methylation analysis reveals gender and age differences in p16INK4a hypermethylation in hepatitis B virus-related hepatocellular carcinoma," Liver International, vol. 32, no. 3, pp. 420-428, 2012.

[24] R. B. Franklin, B. A. Levy, J. Zou et al., "ZIP14 zinc transporter downregulation and zinc depletion in the development and progression of hepatocellular cancer," Journal of Gastrointestinal Cancer, vol. 43, no. 2, pp. 249-257, 2012.

[25] R. B. Gill, A. Day, A. Barstow, G. Zaman, C. Chenu, and G. K. Dhoot, "Mammalian Sulf1 RNA alternative splicing and its significance to tumour growth regulation," Tumour Biology, vol. 33, no. 5, pp. 1669-1680, 2012.

[26] N. T. Snider, P. J. Altshuler, S. Wan, T. H. Welling, J. Cavalcoli, and M. B. Omary, "Alternative splicing of human NT5E in cirrhosis and hepatocellular carcinoma produces a negative regulator of ecto-5'-nucleotidase (CD73)," Molecular Biology of the Cell, vol. 25, no. 25, pp. 4024-4033, 2014.

[27] Y. L. Choi, S. H. Park, J. J. Jang, and C. K. Park, "Expression of the G1-S modulators in hepatitis B virus-related hepatocellular carcinoma and dysplastic nodule: association of cyclin D1 and p53 proteins with the progression of hepatocellular carcinoma," Journal of Korean Medical Science, vol. 16, no. 4, pp. 424-432, 2001.

[28] V. Tripathi, Z. Shen, A. Chakraborty et al., "Long noncoding RNA MALAT1 controls cell cycle progression by regulating the expression of oncogenic transcription factor B-MYB," PLoS Genetics, vol. 9, no. 3, article e1003368, 2013.

[29] L. Wang, J. Zhang, L. Wan, X. Zhou, Z. Wang, and W. Wei, "Targeting Cdc20 as a novel cancer therapeutic strategy," Pharmacology \& Therapeutics, vol. 151, pp. 141-151, 2015.

[30] M. Shi, W. Q. Dai, R. R. Jia et al., "APC ${ }^{\mathrm{CDC} 20}$-mediated degradation of PHD3 stabilizes HIF-1a and promotes tumorigenesis in hepatocellular carcinoma," Cancer Letters, vol. 496, pp. 144-155, 2021.

[31] T. Nakajima, K. Yasui, K. Zen et al., "Activation of B-Myb by E2F1 in hepatocellular carcinoma," Hepatology Research, vol. 38, no. 9, pp. 886-895, 2008.

[32] A. Watanuki, S. Ohwada, T. Fukusato et al., "Prognostic significance of DNA topoisomerase IIalpha expression in human hepatocellular carcinoma," Anticancer Research, vol. 22, no. 2B, pp. 1113-1119, 2002.

[33] H. H. Chan, T. H. Chu, H. F. Chien et al., "Rapid induction of orthotopic hepatocellular carcinoma in immune-competent rats by non-invasive ultrasound-guided cells implantation," BMC Gastroenterology, vol. 10, no. 1, 2010.

[34] N. Wang, M. Zhu, S. W. Tsao, K. Man, Z. Zhang, and Y. Feng, "MiR-23a-mediated inhibition of topoisomerase 1 expression potentiates cell response to etoposide in human hepatocellular carcinoma," Molecular Cancer, vol. 12, no. 1, p. 119, 2013. 
[35] N. Wong, W. Yeo, W.-L. Wong et al., “TOP2A overexpression in hepatocellular carcinoma correlates with early age onset, shorter patients survival and chemoresistance," International Journal of Cancer, vol. 124, no. 3, pp. 644-652.

[36] H. J. Kim, H. J. Kim, S. B. Lee et al., "A proposal for a new classification of T4 breast cancer as stage IIIC: a report from the Korean Breast Cancer Society," Breast Cancer Research and Treatment, vol. 153, no. 1, pp. 153-160, 2015.

[37] R. Panvichian, A. Tantiwetrueangdet, N. Angkathunyakul, and S. Leelaudomlipi, "TOP2A amplification and overexpression in hepatocellular carcinoma tissues," BioMed Research International, vol. 2015, Article ID 381602, 8 pages, 2015.

[38] M. J. Costa, C. L. Hansen, J. A. Holden, and D. Guinee Jr., "Topoisomerase II alpha: prognostic predictor and cell cycle marker in surface epithelial neoplasms of the ovary and peritoneum," International Journal of Gynecological Pathology, vol. 19, no. 3, pp. 248-257, 2000.

[39] A. M. Dingemans, M. A. Witlox, R. A. Stallaert, P. van der Valk, P. E. Postmus, and G. Giaccone, "Expression of DNA topoisomerase IIalpha and topoisomerase IIbeta genes predicts survival and response to chemotherapy in patients with small cell lung cancer," Clinical Cancer Research, vol. 5, no. 8, pp. 2048-2058, 1999.

[40] A. C. Lazaris, N. G. Kavantzas, H. S. Zorzos, N. V. Tsavaris, and P. S. Davaris, "Markers of drug resistance in relapsing colon cancer," Journal of Cancer Research and Clinical Oncology, vol. 128, no. 2, pp. 114-118, 2002.

[41] A. M. Bashour and G. S. Bloom, " $58 \mathrm{~K}$, a microtubule-binding Golgi protein, is a formiminotransferase cyclodeaminase," The Journal of Biological Chemistry, vol. 273, no. 31, pp. 1961219617, 1998.

[42] D. Kohls, T. Sulea, E. O. Purisima, R. E. MacKenzie, and A. Vrielink, "The crystal structure of the formiminotransferase domain of formiminotransferase-cyclodeaminase: implications for substrate channeling in a bifunctional enzyme," Structure, vol. 8, no. 1, pp. 35-46, 2000.

[43] H. Hagiwara, Y. Tajika, T. Matsuzaki, T. Suzuki, T. Aoki, and K. Takata, "Localization of Golgi $58 \mathrm{~K}$ protein (formiminotransferase cyclodeaminase) to the centrosome," Histochemistry and Cell Biology, vol. 126, no. 2, pp. 251-259, 2006.

[44] J. Chen, Z. Chen, Z. Huang, H. Yu, Y. Li, and W. Huang, "Formiminotransferase cyclodeaminase suppresses hepatocellular carcinoma by modulating cell apoptosis, DNA damage, and phosphatidylinositol 3-kinases (PI3K)/Akt signaling pathway," Medical Science Monitor, vol. 25, pp. 4474-4484, 2019.

[45] M. Seimiya, T. Tomonaga, K. Matsushita et al., "Identification of novel immunohistochemical tumor markers for primary hepatocellular carcinoma; clathrin heavy chain and formiminotransferase cyclodeaminase," Hepatology, vol. 48, no. 2, pp. 519-530.

[46] L. Zhuang, Z. Yang, and Z. Meng, "Upregulation of BUB1B, CCNB1, CDC7, CDC20, and MCM3 in tumor tissues predicted worse overall survival and disease-free survival in hepatocellular carcinoma patients," BioMed Research International, vol. 2018, Article ID 7897346, 8 pages, 2018.

[47] J. Y. Liang, D. S. Wang, H. C. Lin et al., “A novel ferroptosisrelated gene signature for overall survival prediction in patients with hepatocellular carcinoma," International Journal of Biological Sciences, vol. 16, no. 13, pp. 2430-2441, 2020.

[48] D. I. Tsilimigras, D. Moris, J. M. Hyer et al., "Hepatocellular carcinoma tumour burden score to stratify prognosis after resection," The British Journal of Surgery, vol. 107, no. 7, pp. 854-864, 2020. 